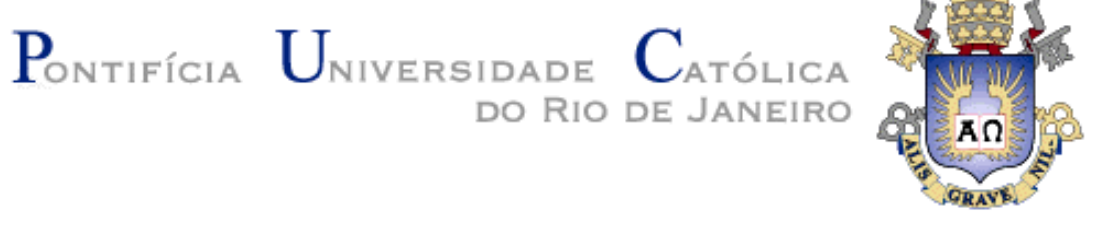

Ingrid Magalhães Tavares da Silva

\title{
Utilização de vácuo em ensaios de laboratório em solo indeformado: carregamento unidimensional e isotrópico
}

\begin{abstract}
Dissertação de Mestrado
Dissertação apresentada como requisito parcial para obtenção do grau de Mestre pelo Programa de PósGraduação em Engenharia Civil do Departamento de Engenharia Civil da PUC-Rio.
\end{abstract}

Orientador: Prof. José Tavares Araruna Jr. Co-Orientador: Prof. Sandro Salvador Sandroni 


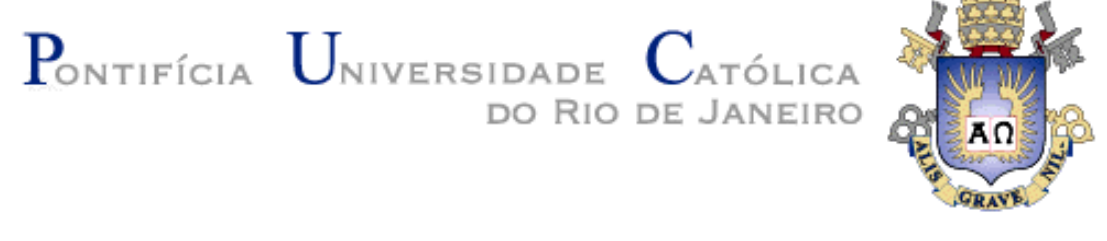

Ingrid Magalhães Tavares da Silva

\author{
Utilização de vácuo em ensaios de \\ laboratório em solo indeformado: \\ carregamento unidimensional e isotrópico
}

Dissertação apresentada como requisito parcial para obtenção do grau de Mestre pelo Programa de PósGraduação em Engenharia Civil do Departamento de Engenharia Civil do Centro Técnico Científico da PUCRio. Aprovada pela Comissão Examinadora abaixo assinada.

\author{
Prof. José Tavares Araruna Jr. \\ Orientador \\ Departamento de Engenharia Civil - PUC-Rio \\ Prof. Sandro Salvador Sandroni \\ Co-Orientador \\ Geoprojetos Ltda \\ Prof. Edgar Odebrecht \\ Universidade do Estado de Santa Catarina \\ Prof. Maria Esther Soares Marques \\ Instituto Militar de Engenharia / RJ
}

Rio de Janeiro, 22 de abril de 2013 
Todos os direitos reservados. É proibida a reprodução total ou parcial do trabalho sem autorização da universidade, do autor e do orientador.

\section{Ingrid Magalhães Tavares da Silva}

Graduou-se em Engenharia Civil na Universidade de Fortaleza (UNIFOR), em Fortaleza - Ceará - Brasil, em 2011.

Ficha Catalográfica

Silva, Ingrid Magalhães Tavares da
Utilização de vácuo em ensaios de laboratório em
solo indeformado : carregamento unidimensional e isotrópico /
Ingrid Magalhães Tavares da Silva ; orientador: Jose Tavares
Araruna Jr. ; co-orientador: Sandro Salvador Sandroni. -
2013. 123 f. : il. (color.); 30 cm
Dissertação (mestrado)-Pontifícia Universidade
Católica do Rio de Janeiro, Departamento de Engenharia
Civil, 2013. $\quad$ Inclui bibliografia
1. Engenharia civil - Teses. 2. Vácuo. 3. Ensaios de
$\quad$ Melhoramento de solos moles. 5.
laboratório.
Adensamento. 6. Compressibilidade. I. Araruna Jr., Jose
Tavares. II. Sandroni, Sandro Salvador. III. Pontifícia
Universidade Católica do Rio de Janeiro. Departamento de
Engenharia Civil. IV. Título.

CDD: 624 
“Tudo é possível àquele que crer"

À minha fonte de inspiração, meu pai, Delto e a minha querida mãe, Bernarda. 


\section{Agradecimentos}

Ao Pai Celeste, o Onipresente e Onisciente, por me dar a força e a energia necessária para cumprir mais uma etapa na minha vida.

Ao meu pai, que me incentivou na Engenharia Geotécnica, e a quem devo muito do que sei e do que aprendi, e à minha amada mãe, meu porto seguro, Bernarda. Meus sinceros agradecimentos àqueles que buscaram sempre me transmitir os ensinamentos da vida.

As minhas queridas irmãs, Fabienne e Chelsea.

A minha família que apesar da distância sempre esteve presente com carinho e confiança.

Ao meu orientador, Jose Tavares Araruna Jr. pelos conceitos transmitidos, sugestões e orientação ao longo de toda pesquisa.

Ao querido professor e orientador, Sandro Sandroni, por todo apoio, mas principalmente por todo tempo dispensado em reuniões, que muito contribuíram na minha formação. Muito obrigada pela sua dedicação, sua paciência, sua confiança e pela amizade.

Ao querido professor Alberto Sayão, a quem muito me ajudou durante toda minha trajetória do mestrado na PUC-Rio, o meu muito obrigado professor pela confiança, pelo incentivo e pela amizade.

À Manuella Galindo, por sua amizade incondicional, pelo apoio e as inúmeras vezes que me ajudou.

Às queridas amigas Juliana Mochel, Simone Peres, Mariana Benessiuti, Stela Martins, Nathalia Passos, Bianca Lima e Jackeline Castañeda pelas contínuas mensagens de apoio, amizade e momentos de descontração.

Aos amigos, Mario Bonillac e Thiago Carnavale, pela amizade e pelo apoio. 
A todos meus amigos da sala 614 e da Pós-Graduação, pelos momentos de alegria e amizade.

Ao corpo docente de Engenharia Civil da PUC-Rio, por todos os importantes ensinamentos transmitidos durante o curso de mestrado.

À empresa Tecnogeo por cederem a bomba de vácuo, necessária ao desenvolvimento deste estudo.

À Monica Moncada pela disposição, ajuda e auxílio fornecido no Laboratório de Geotecnia. Aos técnicos do laboratório, Deivid, Amaury, Rogério, Alex e Josué pelo apoio para realizar os ensaios.

Ao $\mathrm{CNPq}$ e à PUC-Rio, pelo apoio financeiro indispensável ao desenvolvimento deste trabalho. 


\section{Resumo}

Silva, Ingrid Magalhães Tavares da; Araruna Jr, José Tavares (Orientador); Sandroni, Sandro Salvador (Co-orientador). Utilização de vácuo em ensaios de laboratório em solo indeformado: carregamento unidimensional e isotrópico. Rio de Janeiro, 2013. 123 p. Dissertação de Mestrado Departamento de Engenharia Civil da Pontifícia Universidade Católica do Rio de Janeiro.

O uso da sobrecarga a vácuo como um método eficiente no tratamento de solo mole é discutido através de ensaios de laboratório, usando solo indeformado. As características do adensamento a vácuo foram investigadas em laboratório através de ensaios edométricos, comparando carregamento com uso de vácuo ou carga convencional. Foram realizados ensaios na câmara triaxial, comparando o uso da pressão de vácuo e pressão atmosférica. Os resultados indicam que: (1) Os resultados dos ensaios edométricos sugerem que a aplicação de vácuo funciona de maneira equivalente ao incremento de carga convencional e induz a valores de recalques maiores quando comparado com a aplicação de uma carga convencional de mesma magnitude. (2) A partir dos resultados dos ensaios triaxiais, verificou-se que o uso da pressão de vácuo acelera a dissipação da poropressão quando comparado ao adensamento com a ação da pressão atmosférica. (3) As características de adensamento do solo não mostram discriminação contra a natureza da pressão de pré-adensamento, se são sob condições de pressão de vácuo, pressão atmosférica ou carregamento convencional.

\section{Palavras-chave:}

Vácuo; ensaios de laboratório; melhoramento de solos moles; adensamento; compressibilidade. 


\section{Abstract}

Silva, Ingrid Magalhães Tavares da; Araruna Jr, José Tavares (Advisor); Sandroni, Sandro Salvador (Co-advisor). Use of vacuum in laboratory tests on undisturbed soil: one-dimensional and isotropic loading. Rio de Janeiro, 2013. 123 p. MSc. Dissertation - Departamento de Engenharia Civil, Pontifícia Universidade Católica do Rio de Janeiro.

The use of vacuum surcharge as an effective technique in the treatment of soft soil is discussed in analysis in laboratory behavior. Laboratoy tests using undisturbed soil were performed to investigate the use of the technique. The characteristics of the vacuum consolidation were studied in the laboratory by oedometer tests comparing loading with the use of vacuum or conventional load. Tests were performed in triaxial chamber comparing the use of vacuum pressure and atmospheric pressure. The results indicate that: (1) The test results of oedometer test indicate that vacuum application operates in a manner equivalent to the conventional incremental load and induces the largest values of settlement when compared with the conventional application of a load of the same magnitude. (2) The results of the triaxial tests, it was found that the use of vacuum pressure accelerates the dissipation of pore pressure when compared with the action of atmospheric pressure. (3) The consolidation characteristics of the soil show no discrimination against the nature of the consolidation pressure, whether they are consolidated under vacuum pressure, atmospheric pressure or loading conventional.

\section{Keywords:}

Vacuum; laboratory tests; soft soils improvement; consolidation; compressibility. 


\section{Sumário}

1 INTRODUÇÃO 18

1.1. Relevância e Justificativa da Pesquisa 18

1.2. Objetivos 19

$\begin{array}{ll}\text { 1.3. Organização do trabalho } & 20\end{array}$

2 REVISÃO BIBLIOGRAFICA 22

2.1. Teoria do Adensamento de Terzaghi 22

2.1.1. Hipóteses Simplificadoras 22

2.1.2. Equação do Adensamento 23

2.1.3. Ensaio de Adensamento Edométrico 30

2.1.4. Ensaio Triaxial 36

2.2. Técnicas Construtivas sobre Solos Moles 38

2.2.1. Substituição Total ou Parcial de Solos Moles 38

2.2.2. Construção em Etapas 39

2.2.3. Aterros sobre Estacas e Colunas $\quad 39$

2.2.4. Drenos Verticais 40

2.2.5. Sobrecarga Convencional 41

2.2.6. Sobrecarga a Vácuo 43

3 PROGRAMA EXPERIMENTAL 56

3.1. Ensaio de Caracterização Geotécnica 57

3.1.1. Granulometria 57

3.1.2. Limites de Consistência 57

3.1.3. Massa Especifica Real dos Grãos 58

3.1.4. Determinação do Teor de Matéria Orgânica 58

3.2. Ensaio de Adensamento Unidimensional 58

3.2.1. Equipamentos e Metodologias do Ensaio 59

3.3. Ensaio de Adensamento Isotrópico 69

3.3.1. Equipamento Triaxial 70 
4 APRESENTAÇÃO E DISCUSÃO DOS RESULTADOS 83

4.1. Ensaios de Caracterização Geotecnia 83

4.1.1. Analise Granulométrica 83

4.2. Ensaio de Adensamento Unidimensional 85

4.3. Ensaio de Adensamento Isotrópico 96

4.3.1. Ensaios com vácuo 97

4.3.2. Ensaio sem vácuo 102

4.3.3. Comportamento da variação volumétrica 107

4.3.4. Comparação da poropressão 110

5 CONCLUSÕES E SUGESTÕES PARA PESQUISAS FUTURAS 113

5.1. Conclusões 113

5.2. Sugestões para pesquisas futuras 115

6 REFERÊNCIAS BIBLIOGRÁFICAS 116

$\begin{array}{ll}\text { ANEXO I EQUAÇÃO DE CALIBRAÇÃO } & 123\end{array}$ 


\section{Índice de Figuras}

Figura 2.1 - Porcentagem de adensamento $U z$ da camada de solo saturado em função da profundidade normalizada $Z$ e do fator tempo Tv para excesso uniforme de poropressão inicial, $\boldsymbol{u} \mathbf{0}$ (Fonte: Lambe e Whitman, 1969)

Figura 2.2 - Grau de adensamento médio de uma camada de solo saturado: (a) incremento da poropressão inicial uniforme; (b) $U$ versus T (Fonte: Lambe e Whitman, 1969) .....................................28

Figura 2.3 -Determinação do coeficiente de adensamento através do Método de Casagrande (Fonte: Casagrande 1936, apud Netto,2006)

Figura 2.4 - Determinação do coeficiente de adensamento através do Método de Taylor (1948).

Figura 2.5 - Índice de vazios versus tensão vertical efetiva (Craig, 2004 apud Romanel 2011). 34

Figura 2.6 - Relação teórica entre a porcentagem de dissipação da poropressão na base da amostra e o fator tempo (Fonte: Bishop e Henkel, 1962) 37

Figura 2.7 - Compensação dos recalques primários através de sobrecarga adicional (Fonte: Adaptado de Johnson, 1970).

Figura 2.8 - Analogia da mola do processo do adensamento com ação da: sobrecarga convencional; (Fonte: Adaptado de Chu e Yan, 2005)...45

Figura 2.9 - Parâmetros do solo (Fonte: Adaptado de Masse et al.,2001)

Figura 2.10 - Analogia da mola do processo de adensamento com ação da: sobrecarga a vácuo. (Fonte: Adaptado de Chu e Yan, 2005) .....46

Figura 2.11 - Variação da poropressão e da tensão efetiva: (a) sobrecarga convencional; (b) sobrecarga a vácuo (Fonte: Adaptado de Chu e Yan, 2005).

Figura 2.12 - Diagrama p' x q' (Fonte: Adaptado de Masse et al.,2001).49 
Figura 2.13 - Sistema de vácuo com membrana (Fonte: Adaptado de Sandroni et al., 2012) 52

Figura 2.14 - Sistema de aplicação de vácuo dreno a dreno (Fonte: Adaptado de Sandroni et al., 2012).... 54

Figura 2.15 - Junção do trecho impermeável com o dreno: (a) dreno fita; (b) dreno estrela (Fonte: Sandroni et al., 2012) 54

Figura 3.1 - Prensa de adensamento tipo Bishop do LGMA da PUC-Rio:

(a) vista de frente $e(b)$ vista lateral. 59

Figura 3.2 - Processo de extração e moldagem da amostra do tubo amostrador..... 61

Figura 3.3 - (a) Célula fabricada de PVC e (b) Célula convencional .......62

Figura 3.4 - Processo de montagem da célula 63

Figura 3.5 - Sistema de vedação da célula de adensamento 64

Figura 3.6 - Célula de adensamento ajustada na prensa com sistema de vedação. 64

Figura 3.7 - (a) Abaixamento do O'ring com auxilio da seringa de plástico e (b) O'ring após abaixamento .65

Figura 3.8 - Topo do Cap preenchido com graxa de vaselina 65

Figura 3.9 - Bomba de vácuo .66

Figura 3.10 - Sistema externo de arrefecimento da água.

Figura 3.11 - Tanque de acrílico com a interface ar/água ........................68

Figura 3.12 - Detalhe do regulador de altura ....................................... 68

Figura 3.13 - Componentes do sistema triaxial utilizado na pesquisa.....71

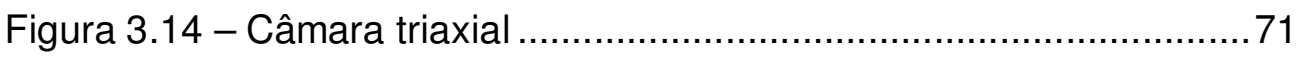

Figura 3.15 - (a) Visão geral do ensaio, (b) Sistema de válvulas na base

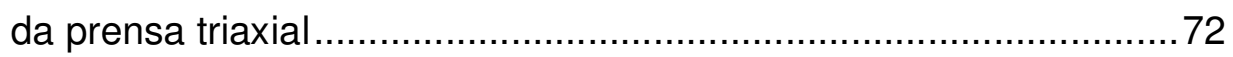

Figura 3.16 - Medidor de Variação Volumétrica ……………….............. 73

Figura 3.17 - Transdutor de pressão durante execução do ensaio ..........75

Figura 3.18 - Sistema de aplicação de pressões ..................................... 75

Figura 3.19 - Sistema central de aquisição de dados .............................76

Figura 3.20 - Válvulas responsáveis pela aplicação da tensão confinante e medição do MVV .79

Figura 3.21 - Válvulas responsáveis pela medição da poropressão no topo da amostra .80 
Figura 3.22 - Válvulas responsáveis pela aplicação da pressão de vácuo ou atmosférica.

Figura 4.1 - Curva granulométrica do solo mole ..................................... 83

Figura 4.2 - Correlação entre umidade e PPI (Fonte: Sandroni, 2012) ...85

Figura 4.3 - Comparação das curvas índice de vazios $x$ tensão vertical efetiva.

Figura 4.4 - Comparação das curvas do sétimo estágio de carregamento

Figura 4.5 - Porcentagem média de adensamento versus fator tempo:

AU-01 90

Figura 4.6 - Porcentagem média de adensamento versus fator tempo: AU02. 90

Figura 4.7 - Coeficiente de adensamento versus tensão vertical efetiva para ensaio $\mathrm{AU}-01$

Figura 4.8 - Coeficiente de adensamento versus tensão vertical efetiva para ensaio AU-02 92

Figura 4.9 - Permeabilidade versus tensão vertical efetiva para ensaio AU-01 .93

Figura 4.10 - Permeabilidade versus tensão vertical efetiva para ensaio AU-02 .93

Figura 4.11 - Coeficiente de Permeabilidade versus tensão vertical efetiva .94

Figura 4.12 - Poropressão versus tempo, amostra $5,5 \mathrm{~cm}$ com vácuo ....99

Figura 4.13 - Poropressão versus tempo, amostra $3,8 \mathrm{~cm}$ com vácuo ....99

Figura 4.14 - Poropressão versus tempo, amostra $2,5 \mathrm{~cm}$ com vácuo .. 100

Figura 4.15 - Grau de adensamento versus Fator Tempo, amostra 5,5 cm com vácuo. 101

Figura 4.16 - Grau de adensamento versus Fator Tempo, amostra 3,8 cm com vácuo. 101

Figura 4.17 - Grau de Adensamento versus Fator Tempo, amostra 2,5 cm com vácuo. 102

Figura 4.18 - Poropressão versus tempo, amostra 5,5 cm sem vácuo .. 104

Figura 4.19 - Poropressão versus tempo, amostra $3,7 \mathrm{~cm}$ sem vácuo .. 104

Figura 4.20 - Poropressão versus tempo, amostra $2,5 \mathrm{~cm}$ sem vácuo .. 105 
Figura 4.21 - Grau de Adensamento versus Fator Tempo, amostra 5,5 cm sem vácuo. 106

Figura 4.22 - Grau de Adensamento versus Fator Tempo, amostra 3,5 cm sem vácuo. 106

Figura 4.23 - Grau de Adensamento versus Fator Tempo, amostra 2,5 cm sem vácuo. 107

Figura 4.24 - Porcentagem de deformação volumétrica versus tempo, amostra de $5,5 \mathrm{~cm}$. 109

Figura 4.25 - Porcentagem de deformação volumétrica versus tempo, amostra de $3,5 \mathrm{~cm}$. 109

Figura 4.26 - Porcentagem de deformação volumétrica versus tempo, amostra de $5,5 \mathrm{~cm}$. 110

Figura 4.27 - Variação da poropressão versus o tempo para amostra de $2,5 \mathrm{~cm}$. 111

Figura 4.28 - Variação da poropressão versus o tempo para amostra de $3,5 \mathrm{~cm}$. 111

Figura 4.29 - Variação da poropressão versus o tempo para amostra de $5,5 \mathrm{~cm}$. 112

Figura A.I.1- Dados e equação da calibração 112 


\section{Índice de Tabelas}

Tabela 2.1 - Avaliação da Porcentagem média de adensamento U 29

Tabela 2.2 - Relação entre o fator tempo $T_{v}$ e a porcentagem de dissipação da poropressão na base da amostra e drenagem a partir do topo (Fonte: Bishop e Henkel, 1962) 37

Tabela 3.1 - Resumo do Programa Experimental 56

Tabela 3.2 - Altura dos ensaios de adensamento isotrópico na câmara triaxial $\quad 70$

Tabela 4.1 - Resumo do ensaio de caracterização 84

Tabela 4.2 - Teor de matéria orgânica 84

Tabela 4.3 - (a) Parâmetros iniciais e (b) Resumo dos resultados do ensaio do adensamento unidimensional AU-01 86

Tabela 4.4 - (a) Parâmetros iniciais e (b) Resumo dos resultados do ensaio do adensamento unidimensional AU-02 87

Tabela 4.5 - Valores dos parâmetros de compressibilidade $\quad 94$

Tabela 4.6 - Índices de compressibilidade para solos moles do Estado do Rio de Janeiro 95

Tabela 4.7 - Resumo dos ensaios realizados $\quad 97$

Tabela 4.8 - Variação de volume dos corpos de prova 108

Figura A.I.1- Curva da calibração . 112 


\title{
Lista de Símbolos
}

\author{
$a_{v} \quad$ Coeficiente de compressibilidade \\ B Parâmetro B de Skempton \\ $\mathrm{C}_{\mathrm{C}} \quad$ índice de compressão \\ $\mathrm{C}_{\mathrm{r}} \quad$ índice de recompressão \\ $\mathrm{C}_{\mathrm{V}} \quad$ coeficiente de adensamento \\ $\mathrm{C}_{\alpha} \quad$ compressão secundária \\ e índice de vazios; \\ Gs densidade relativa dos grãos \\ $H_{d} \quad$ distância de drenagem \\ $k_{v} \quad$ coeficiente de permeabilidade vertical \\ Ko coeficiente de empuxo no Repouso \\ $t \quad$ tempo; \\ $T_{v} \quad$ fator tempo \\ $m_{v} \quad$ coeficiente de variação volumétrica \\ $\mathrm{Pa} \quad$ pressão atmosférica \\ Psc carga provisória \\ $\mathrm{P}_{\mathrm{PE}} \quad$ carga permanente \\ $\Delta \mathrm{H}_{\mathrm{PE}} \quad$ variação do recalque devido a carga permanente \\ $\Delta \mathrm{H}_{\mathrm{SC}} \quad$ variação do recalque devido a carga provisória \\ S grau de saturação \\ Uv porcentagem de adensamento \\ $\cup \quad$ porcentagem média de adensamento \\ $u_{e} \quad$ excesso de poropressão no tempo t \\ $\overline{u_{*}} \quad$ excesso de poropressão média na camada \\ $u_{0} \quad$ excesso de poropressão inicial \\ w umidade \\ z distância vertical entre um ponto e a superfície de aplicação \\ do carregamento
}

$\gamma_{\text {nat }}-\quad$ peso específico natural do solo 
$\gamma_{w}-$ peso específico da água

$\Delta e$ - variação do índice de vazios

$\Delta \mathrm{p}$ - incremento de pressão

$\Delta \sigma^{\prime}{ }_{v}$ - variação da tensão vertical efetiva

$\Delta \sigma_{G}-$ acréscimo de tensão confinante aplicado

$\Delta u-$ excesso de poropressão gerado

$\sigma_{c}$ - tensão confinante

$\sigma^{\prime}{ }_{0}$ - tensão efetiva inicial

$\varepsilon$ - deformação

$\varepsilon_{f}-$ deformação final

$\sigma^{\prime} \boldsymbol{h}$ - tensão horizontal efetiva

$\sigma^{\prime} v$ - tensão vertical efetiva 